\title{
Exploiting Monotonicity Constraints in Active Learning for Ordinal Classification
}

\author{
Pieter Soons \\ Universiteit Utrecht \\ Utrecht, The Netherlands \\ e-mail: p.s@live.nl
}

\author{
Ad Feelders \\ Universiteit Utrecht \\ Utrecht, The Netherlands \\ e-mail: A.J.Feelders@uu.nl
}

\begin{abstract}
We consider ordinal classification and instance ranking problems where each attribute is known to have an increasing or decreasing relation with the class label or rank. For example, it stands to reason that the number of query terms occurring in a document has a positive influence on its relevance to the query. We aim to exploit such monotonicity constraints by using labeled attribute vectors to draw conclusions about the class labels of order related unlabeled ones. Assuming we have a pool of unlabeled attribute vectors, and an oracle that can be queried for class labels, the central problem is to choose a query point whose label is expected to provide the most information. We evaluate different query strategies by comparing the number of inferred labels after some limited number of queries, as well as by comparing the prediction errors of models trained on the points whose labels have been determined so far. We present an efficient algorithm to determine the query point preferred by the well-known active learning strategy generalized binary search. This algorithm can be applied to binary classification on incomplete matrix orders. For non-binary classification, we propose to include attribute vectors in the training set whose class labels have not been uniquely determined yet. We perform experiments on artificial and real data.
\end{abstract}

\section{Introduction}

In some learning problems, we know for each attribute whether it has a positive or negative influence on the response variable. For example, it stands to reason that the number of query terms occurring in a document has a positive influence on its relevance to the query. In the context of ordinal classification or instance ranking problems, prior knowledge of this kind provides us with the useful constraint that if $a$ scores better (not worse) on all attributes than $b$, then the class label of $a$ can't be lower than the class label of $b$. For example, if $a$ and $b$ apply for a loan, and $a$ scores better than $b$ on all criteria, then it would be rather surprising if the application of $b$ is accepted, but $a$ 's is rejected. Quite some work has been done on the development of algorithms that enforce such monotonicity constraints on the models they produce, see for example $[6,1]$.

We propose to use this prior knowledge in the context of active learning to augment the training sample with data points whose labels can be (partially) inferred from those of order related points whose label was provided explicitly by an oracle. We consider the pool-based active learning setting where we initially have a pool of unlabeled data points, and we can ask an oracle for the label of (a limited number of) data points. The central problem is to decide which data point to submit to the oracle. Generally speaking, we would like to submit one for which we expect it will enable us to draw conclusions about the labels of many other points. The problem is how to formalize this general notion, and to find efficient algorithms to determine the corresponding query points.

This paper builds on the work of Barile and Feelders in [3]. The most important extensions are:

1. For binary classification, we have developed an efficient algorithm to evaluate the well-known active learning heuristic generalized binary search (see section 3.1). This algorithm can be used in case the order on the data points is an incomplete matrix order, or 2-dimensional order.

2. For non-binary classification, we have developed a way to include data points into the training sample whose label has not been uniquely determined yet (see section 3.2).

We compare the performance of these new algorithms with those of [3] in section 5. We have furthermore developed an instance of the Propp-Wilson algorithm with sandwiching that enables us to draw a random monotone classification over a given partial order. This algorithm is used to generate artificial data sets for 
the evaluation of our active learning algorithms, and is described in section 4 .

We first discuss some preliminaries however.

\section{Preliminaries}

Let $\mathcal{X}$ be an attribute space $\mathcal{X}=\mathcal{X}^{1} \times \mathcal{X}^{2} \times \ldots \times \mathcal{X}^{p}$ consisting of vectors $x=\left(x^{1}, x^{2}, \ldots, x^{p}\right)$ of values on $p$ attributes. We assume that each attribute takes values in a linearly ordered set $\mathcal{X}^{h}$. The partial ordering $\preceq$ on $\mathcal{X}$ is the ordering induced by the order relations on its coordinates $\mathcal{X}^{h}$ :

$$
x_{i} \preceq x_{j} \Leftrightarrow \forall h=1, \ldots, p: x_{i}^{h} \leq x_{j}^{h} .
$$

Likewise, let $\mathcal{Y}$ be a finite linearly ordered set of classes. Without loss of generality, we assume that $\mathcal{Y}=\{1,2, \ldots, k\}$ where $k$ is the number of classes.

We have available an unlabeled training sample $X=\left\{x_{1}, \ldots, x_{n}\right\}$ with $X \subset \mathcal{X}$. The monotonicity assumption states that

$$
x_{i} \preceq x_{j} \Rightarrow y_{i} \leq y_{j} .
$$

This constraint expresses the prior knowledge that the class label is increasing in each of the attributes, and it is the basis of all inference. If the class label is decreasing rather than increasing in an attribute, we can simply invert the values of the attribute. A classification that satisfies (2.1) is called a monotone classification. The degree of comparability of $X$ is the fraction of pairs of data points $\left(x_{i}, x_{j}\right)$ with $x_{i} \preceq x_{j}$ or $x_{j} \preceq x_{i}$.

A lower set $L$ is a subset of $X$ that contains the downward closure of all its elements:

$$
x_{i} \in L, x_{j} \preceq x_{i} \Rightarrow x_{j} \in L .
$$

Likewise, an upper set $U$ of $X$ contains the upward closure of all its elements. The downset $\downarrow\left(x_{i}\right)$ of $x_{i}$ contains all elements of $X$ that are smaller than or equal to $x_{i}$ :

$$
\downarrow\left(x_{i}\right)=\left\{x_{j} \in X: x_{j} \preceq x_{i}\right\} .
$$

The upset $\uparrow\left(x_{i}\right)$ of $x_{i}$ is defined analogously. We use $d\left(x_{i}\right)=\left|\downarrow\left(x_{i}\right)\right|$ to denote the size of the downset of $x_{i}$, and $u\left(x_{i}\right)$ to denote the size of its upset.

\section{Active Learning}

We consider the setting of pool-based active learning. A fundamental assumption in this paper is that the oracle always produces monotone class labels, that is, class labels that satisfy constraint (2.1). We first consider the case of binary classification; section 3.2 discusses the non-binary case.
3.1 Binary Classification Dasgupta [4] analyzes an active learning strategy that can be summarized as follows. Let $H$ denote a hypothesis class (e.g. the class of monotone functions), and let $\widehat{H}$ denote the effective hypothesis class for a given sample of unlabeled points $X=\left\{x_{1}, \ldots, x_{n}\right\}$ (e.g. the class of monotone functions on $X)$. Let $\pi$ denote a probability distribution over $\widehat{H}$. The objective is to determine the unique $h \in \widehat{H}$ that is consistent with all the hidden labels, by querying as few of them as possible. Let $S \subseteq \widehat{H}$ be the set of hypotheses that is consistent with the labels queried so far. For each unlabeled $x_{i}$, let $S_{i}^{+}$be the hypotheses which label $x_{i}$ positive and $S_{i}^{-}$the ones which label it negative. The proposed strategy, which we will henceforth refer to as generalized binary search (or GBS for short) is to pick the $x_{i}$ for which these sets are most nearly equal in probability, that is, the $x_{i}$ for which $\pi\left(S_{i}^{-}\right)$is closest to $\frac{1}{2}$. Another (equivalent) interpretation is that the query point whose class distribution has the highest entropy is selected. Dasgupta [4] shows that if the optimal query strategy (that is, the one that requires the fewest queries in expectation to determine $h$ ) requires $Q^{*}$ queries in expectation, then the expected number of queries needed by GBS is at most $4 Q^{*} \ln 1 /\left(\min _{h} \pi(h)\right)$. In particular, if $\pi$ is the uniform distribution, it requires at most $4 \ln |\widehat{H}| Q^{*}$ queries in expectation. Henceforth we assume that $\pi$ is uniform.

Let us consider how we can implement GBS for our problem. Let $\widehat{H}$ be the collection of all monotone binary classifications of the pool of unlabeled points $X$. Note that there is a one-to-one correspondence between monotone binary classifications on $(X, \preceq)$, and lower sets of $(X, \preceq)$ : given a lower set $L \subseteq X$, the classification function

$$
f(x)= \begin{cases}1 & \text { if } x \in L \\ 2 & \text { otherwise }\end{cases}
$$

is monotone, and vice versa, given a monotone classification $f$, the set

$$
L=\{x \in X: f(x)=1\}
$$

is a lower set of $X$. Hence, to determine the size of $S_{i}^{-}$we need to count the number of lower sets that include $x_{i}$. Unfortunately, counting the lower sets of a partial order is \#P complete [8]. For this reason, Barile and Feelders [3] proposed the easy-to-compute GREEDY heuristic

$$
x^{*}=\arg \max _{x \in X} \min \{d(x), u(x)\},
$$

where $x^{*}$ denotes the selected query point. This heuristic maximizes the number of labels we can infer in the 
worst case, and only requires the computation of the size of the upset and downset of each potential query point.

We note however that for certain types of partial order the problem of counting lower sets is tractable. For example, the number of lower sets of a chain of length $n$ is $n+1$, and both GBS and GREEDY query the point in the middle of the chain. This is known to be optimal, and the number of queries required to determine all labels is $1+\log _{2} n$. A somewhat less restrictive order is the matrix order. We get an $m \times n$ matrix order for example if we have two attributes $X_{1}$ and $X_{2}$, with domain sizes $\left|\mathcal{X}^{1}\right|=m$ and $\left|\mathcal{X}^{2}\right|=n$. We have developed an efficient dynamic programming algorithm to count the lower sets for this type of order. We describe this algorithm in the remainder of this section (a proof of its correctness is given in [9]). Since it might be the case that not all possible vectors $(i, j)$ $(i=1, \ldots, m, j=1, \ldots, n)$ are observed in the sample, we must take into account the possibility that some of the cells of the matrix are "empty". We count the lower sets of the partial order defined by: $(i, j) \preceq(k, l) \Leftrightarrow i \leq$ $k$ and $j \leq l$. Let $D_{i, j}$ denote the number of lower sets of the part of the matrix with row $\leq i$ and column $\geq j$. To count the lower sets of the whole matrix, we can use the following recursion: $D_{i, j}=$

$$
\begin{cases}D_{i-1, j}+D_{i, j+1}-D_{i-1, j+1} & \text { if }(i, j) \text { is empty } \\ D_{i-1, j}+D_{i, j+1} & \text { otherwise }\end{cases}
$$

All we need to kick start the process is to determine the counts for row 1 and column $n$, but this is straightforward since the corresponding blocks are linear orders. If cell $(1, n)$ happens to be empty we enter a count of 1 since the empty set is a lower set.

Consider the example of the $4 \times 4$ matrix given in Table 1(a). The grey cells are empty. To compute $D(3,2)$ for example, we add $D(2,2)$ and $D(3,3)$, and because $(3,2)$ is an empty cell, we subtract $D(2,3)$ giving a count of 8 lower sets. We read in cell $(4,1)$ that this matrix order has 22 lower sets (monotone binary classifications) in total.

This establishes the total number of lower sets of a matrix order, but we need to count the number of lower sets that include $x$. As it turns out this can be done with a few extra steps. The number of lower sets of $X$ that include $x$ is equal to the number of lower sets of $X \backslash \downarrow x$. This number cannot be read off immediately from $D$, except for the cells in the top row. For example, the downset of cell $(4,1)$ is the entire first column of the matrix, so the required count can be found in cell $(4,2)$. To compute the counts for cells not in the top row, we first define a matrix $E$ which is constructed in a similar fashion as $D$, but working in the opposite direction, i.e.

\begin{tabular}{l|cc||c|cc}
\hline cell & $C(i, j)$ & $P(y=1)$ & cell & $C(i, j)$ & $P(y=1)$ \\
\hline$(1,1)$ & 21 & .95 & $(2,3)$ & 13 & .59 \\
$(2,1)$ & 18 & .82 & $(3,3)$ & 9 & .41 \\
$(1,2)$ & 19 & .86 & $(4,3)$ & 3 & .14 \\
$(4,2)$ & 10 & .45 & $(2,4)$ & 8 & .36 \\
$(1,3)$ & 16 & .73 & $(3,4)$ & 3 & .14 \\
\hline
\end{tabular}

Table 2: Computations necessary for evaluating GBS: for every non-empty cell, the number of lower sets $C(i, j)$ that contain that cell, and the corresponding probability that this cell has class label 1 are given. GBS selects the query point $(4,2)$

from the upper-left cell to the bottom-right cell. Cell $(i, j)$ of $E$ contains a count of the lower sets of the block extending from the upper-left cell to cell $(i, j)$. The total count will now appear in the bottom-right cell of $E$ (see Table 1(b)). Finally we define the matrix $C$ where $C(i, j)$ contains the number of lower sets that include cell $(i, j) . C_{i, j}=$

$$
\begin{cases}D_{i, j+1} & \text { if } i=m \\ C_{i+1, j}+D_{i, j+1} \times\left(E_{i+1, j}-E_{i+2, j}\right) & \text { otherwise }\end{cases}
$$

where, all "out of bounds" cells should be taken to contain a count of 1 . The matrix $C$ for our example is given in Table 1(c). We can read from it for example that the number of lower sets that include $(2,3)$ is 13 . To determine the complexity of this algorithm, we note that we need to perform just a few elementary computations for each cell in the matrix, so the complexity is $O(m n)$, which is proportional to the number of observed data vectors.

Let us compute which query point would be selected by the GBS heuristic. In Table 2 we have listed the data required for its evaluation. Here we see that the class label distribution for the cell $(4,2)$ has the highest entropy: in $45 \%$ (10 out of 22 ) of all monotone classifications it has label 1, and in 55\% it has label 2. Since the entropy for all other query points is smaller, GBS will select $(4,2)$. The necessary computations to evaluate the GREEDY heuristic are summarized in Table 3. If we query for the label of cell $(2,3)$ we can determine the label of 5 cells, regardless of whether the true label is 1 or 2 . All other cells have a lower worst case outcome, so the cell $(2,3)$ is selected by GREEDY.

Finally, we note that after querying for the label of $x$, the labels of either the downset (if the label is 1 ) or the upset (if the label is 2) of $x$ are determined due to the monotonicity constraint, and hence the corresponding vectors can be removed from the partial order. The remaining order is again an incomplete matrix order, 


\begin{tabular}{l|cccc}
4 & 22 & 18 & 10 & 3 \\
3 & 12 & 8 & 7 & 3 \\
2 & 9 & 5 & 4 & 2 \\
\cline { 2 - 4 } & 4 & 3 & 2 & 1 \\
\hline & 1 & 2 & 3 & 4
\end{tabular}

(a) Matrix $D$

\begin{tabular}{c|cccc}
4 & 1 & 2 & 3 & 3 \\
3 & 1 & 2 & 5 & 8 \\
2 & 2 & 3 & 8 & 16 \\
1 & 3 & 6 & 14 & 22 \\
\cline { 2 - 5 } & 1 & 2 & 3 & 4
\end{tabular}

(b) Matrix $E$

\begin{tabular}{c|cccc|}
4 & 18 & 10 & 3 & 1 \\
3 & 18 & 17 & 9 & 3 \\
2 & 18 & 17 & 13 & 8 \\
1 & 21 & 19 & 16 & 16 \\
\cline { 2 - 4 } & 1 & 2 & 3 & 4
\end{tabular}

(c) Matrix $C$

Table 1: Counts for an example $4 \times 4$ matrix order. The grey cells correspond to attribute vectors that are not observed in the sample. The cells are numbered so as to correspond with the ordering in the plane, e.g., the smallest element $(1,1)$ is the bottom-left cell.

\begin{tabular}{|l|ccc||c|ccc|}
\hline cell & $d$ & $u$ & min & cell & $d$ & $u$ & $\min$ \\
\hline$(1,1)$ & 1 & 10 & 1 & $(2,3)$ & 5 & 5 & 5 \\
$(2,1)$ & 2 & 7 & 2 & $(3,3)$ & 6 & 3 & 3 \\
$(1,2)$ & 2 & 8 & 2 & $(4,3)$ & 8 & 1 & 1 \\
$(4,2)$ & 4 & 2 & 2 & $(2,4)$ & 6 & 2 & 2 \\
$(1,3)$ & 3 & 6 & 3 & $(3,4)$ & 8 & 1 & 1 \\
\hline
\end{tabular}

Table 3: Computations necessary for evaluating GREEDY: for every non-empty cell, the size $d$ of its downset, the size $u$ of its upset, and the minimum of them is given. GREEDY selects the query point $(2,3)$.

so the next query point can be determined in the same way.

3.2 Non-binary Classification In the binary case, the GBS heuristic selected the query point whose class distribution had the largest entropy. This principle can be extended to the non-binary case, but it requires even more complex computations, as was to be expected.

Given a nested sequence $L_{1} \subseteq L_{2} \subseteq \ldots \subseteq L_{k-1}$ of lower sets of $X$, the classification function:

$$
f(x)=\left\{\begin{array}{ll}
j & \text { if } x \in L_{j} \backslash \bigcup_{i<j} L_{i}, \quad j=1, \ldots, k-1 \\
k & \text { otherwise }
\end{array},\right.
$$

is monotone. Conversely, given a monotone classification function $f(x), x \in X$, define $L_{i}=\{x \in X: f(x) \leq$ $i\}, i=1, \ldots, k-1$. The sequence $L_{1}, \ldots, L_{k-1}$ is a nested sequence of lower sets of $X$. Hence, to count the number of monotone classifications on $X$, we can count the number of nested sequences of lower sets of $X$ instead. We have not been able to find an efficient way to do this even for a matrix order.

Therefore we extend the GREEDY heuristic to nonbinary classification problems, as proposed in [3]. It doesn't make much sense now to count the number of class labels that can be inferred in the worst case, as that number will typically be zero (or one if you include the the label of the query point itself) for all potential query points. Hence, we switch to counting the number of labels that can be eliminated in the worst case instead.

Let $\left[\ell_{i}, h_{i}\right]$ denote the interval of possible labels for $x_{i}$. Initially we have $\ell_{i}=1$ and $h_{i}=k$ for all $i$, but as we learn the labels of points, using the monotonicity constraint these bounds are adjusted. Let $N\left(x_{i}, y\right)$ denote the number of values we can eliminate when $y_{i}=y$. We have

$$
N\left(x_{i}, y\right)=\sum_{x_{j} \in \downarrow\left(x_{i}\right)}\left(h_{j}-y\right)_{+}+\sum_{x_{j} \in \uparrow\left(x_{i}\right)}\left(y-\ell_{j}\right)_{+},
$$

where $z_{+}=\max (0, z)$. Maximizing the worst case we select the query point

$$
x^{*}=\arg \max _{x_{i} \in X} \min _{y \in\left[\ell_{i}, h_{i}\right]}\left\{N\left(x_{i}, y\right)\right\} .
$$

As the number of class labels $k$ increases, it becomes more and more difficult to infer unique class labels for points that have not been queried. This means that it becomes very difficult to infer a sizeable training set from just a limited number of queries. On the other hand there may be quite a few points whose set of possible labels has been reduced considerably by inference from the query points. If we only include points whose label has been uniquely determined in the training set, then we completely ignore this potentially valuable information. Therefore we propose to exploit partial label information as follows. Let $x_{i}$ be an attribute vector whose label set has been reduced to the interval $\left[\ell_{i}, h_{i}\right]$. The size of this interval is $s_{i}=h_{i}-\ell_{i}+1$. We add $s_{i}$ copies of $x_{i}$ to the training set, with respective labels $\ell_{i}, \ldots, h_{i}$. Each copy gets the weight $s_{i}^{-1}$.

The question remains how many labels have to be eliminated before we decide to include a point in the training sample. This is determined by the value of the parameter $a \in[0,1]$, where $x_{i}$ is included if $s_{i} \leq a \cdot k$. So if $a=\frac{1}{2}$, we include a point if at least half of its possible labels have been eliminated. Algorithm 1 shows how we use the partial label information to predict the class of a new data point $z$ with a $K$-nearest neighbor classifier. In the call, $T$ denotes the training sample. The call to GetNearestNeighbors returns 
the $K$ nearest neighbors of $z$ in $T$, where only attribute vectors $x_{i}$ in $T$ with $s_{i} \leq a \cdot k$ are considered. In predicting the label of $z$, the weight of a training point is inversely proportional to its distance to $z$. In case a training point has multiple possible labels, the weight is distributed evenly over the different labels. To get the final prediction, the weighted average is rounded to the nearest integer. We will use Algorithm 1 to assess the quality of a query strategy by measuring the prediction error on an independent test sample of this classifier, using a training sample $T$ that has been constructed with that query strategy. It should be noted that the classifier is merely an instrument to assess the quality of a query strategy, so we have not attempted to find the best classifier. The partial label information can also be used by other classification algorithms that allow the specification of case weights in training.

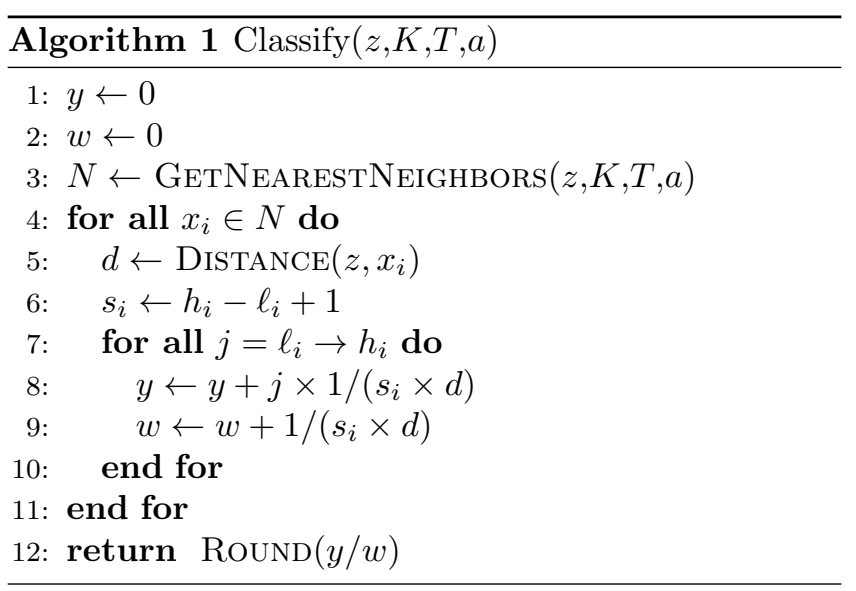

\section{Generating random monotone functions}

In this section we present an algorithm to uniformly sample a random monotone classification function with (at most) $k$ labels, defined on a given partial order $(X, \preceq)$. This algorithm will be used to generate artificial data sets for the evaluation of the proposed active learning heuristics. It is an instance of the ProppWilson algorithm [7] with sandwiching.

Recall that we established a one-to-one correspondence between nested sequences of lower sets and monotone classification functions in section 3.2. Let $\mathcal{S}$ denote the collection of all nested sequences of lower sets on $(X, \preceq)$. We define an order $(\mathcal{S}, \preceq \mathcal{S})$ on the elements $S$ of $\mathcal{S}$ as follows. Let $S_{1}=L_{1}^{S_{1}}, \ldots, L_{k-1}^{S_{1}}$ and $S_{2}=L_{1}^{S_{2}}, \ldots, L_{k-1}^{S_{2}}$ denote two nested sequences of lower sets. The order is defined as:

$$
S_{1} \preceq \mathcal{S} S_{2} \Leftrightarrow \forall i=1, \ldots, k-1: L_{i}^{S_{2}} \subseteq L_{i}^{S_{1}} .
$$

Note that this order has a least element $S_{\perp}=\forall i=$
$1, \ldots, k-1: L_{i}=X$ (all elements of $X$ get the smallest label), and a greatest element $S^{\top}=\forall i=1, \ldots, k-1$ : $L_{i}=\varnothing$ (all elements of $X$ get the largest label).

We set up a number of Markov chains that have $\mathcal{S}$ as their state space. Let $\phi_{t}(S, u)$ denote the update function, where $S \in \mathcal{S}$ is the current state and $u$ is a uniformly distributed random number in the interval $(0,1)$. The chains are coupled, that is, the same random number $u_{t}$ is used by all chains. Therefore, once two chains "meet" in the same state, they become "stuck forever". Such chains are said to have coalesced. Now suppose the update function is monotone, that is

$$
S_{1} \preceq_{\mathcal{S}} S_{2} \Rightarrow \phi_{t}\left(S_{1}, u_{t}\right) \preceq_{\mathcal{S}} \phi_{t}\left(S_{2}, u_{t}\right) .
$$

If we have coupled chains that start in $S_{1}, S_{2}$ and $S_{3}$ with $S_{1} \preceq_{\mathcal{S}} S_{2} \preceq_{\mathcal{S}} S_{3}$, and at time $t$, the chains started at $S_{1}$ and $S_{3}$ have coalesced, then the monotone update function guarantees that the chain that started in $S_{2}$ has the same value. If in the partial order we have a least element and a greatest element, then we only need two chains, started in these two states. Once these two chains converge, all chains started in other states would have converged to the same value.

Hence, for the algorithm to work, it is essential to use a monotone update function. In [9] we present the update function, and prove that it is monotone.

To summarize the algorithm: we use two coupled chains, started in $S_{\perp}$ and $S^{\top}$ and run them "in the past" from time $-T$ to -1 . If after this time, the chains have coalesced, we use their value as our sample. If they have not coalesced, we increase the amount of time and run the chains again. Once the chains have converged at $t=0$ for some $T$, we would still get the same answer if we had started the chains even earlier. So in effect, we get a sample from a chain that has been run for an infinite amount of time. Propp and Wilson [7] show that by running this Markov chain we generate a uniform random element from $\mathcal{S}$.

\section{Experiments}

To evaluate the different query strategies, we compare their performance on artificial and real data sets. We would like to stress that we have not attempted to systematically optimize the parameters of the classification model (nearest neighbor), because it is merely instrumental in measuring the quality of the different query strategies. The value of $K$ for the $K$-nearest neighbor algorithm was fixed at 5 because preliminary experiments demonstrated that this value worked quite well. Throughout the experiments we fixed the ambiguity parameter $a$ at $\frac{1}{2}$, as it makes sense to only use the partial label information if more than half of the possible labels have been ruled out. 
5.1 Experiments on Artificial Data We generate artificial data in two different ways, one specifically intended for the incomplete matrix order, and one more general method. To generate data for the matrix order, we pick a value $m$ for the number of rows and columns of the matrix (for simplicity we assume the matrix is square). Furthermore, we choose a value $d \in[0,1]$ which denotes the fraction of observed cells. Given an incomplete matrix order generated in this way, we use the algorithm described in section 4 to generate a random monotone binary classification on this order. We only consider binary classifications on matrix orders, so that we can use the efficient lower sets counting algorithm of section 3.1 to compute the GBS query point.

Table 4 and Figure 1 summarize some of our findings. In Table 4 we see for example that the GREEDY heuristic only needs 12 queries to infer $80 \%$ of the labels in the training set consisting of two thirds of $n=1250$ is 833 attribute vectors. With respect to the number of inferred labels, GREEDY dominates GBS for a long time but is taken over by it at the very end: when it comes to classifying $100 \%$ of the training sample GBS requires fewer queries. Note that in the beginning we can infer many labels from just a few queries, but to obtain the last $20 \%$ of labels requires relatively many queries. For example, for $m=50$ and $d=0.2$, GBS requires 13.4 queries on average to infer $80 \%$ of the labels, but to obtain the last $20 \%$ an additional 28.6 queries are needed. Likely this is because towards the end, the partial order disintegrates into a number of smaller disconnected components, which limits the scope for inference. Figure 1 gives the detailed results for different query strategies for $k=2, m=50$, and $d=0.5$. The RANDOM ( $\mathrm{R}$ in Figure 1) strategy picks an unlabeled point at random but does use the monotonicity constraint to infer the labels of other points. The R-NOINF strategy doesn't even use the monotonicity constraint, so its training sample consists only of the points that were explicitly labeled by the oracle. It was merely included to show the benefit of the constraint. The line labeled ALL gives the results obtained when the complete labeled training sample is used, and provides a natural upper bound for performance. The results of GBS are given by the line labeled MATRIX in Figure 1.

To study the behavior in case there are more than two class labels, we generate data from a bivariate normal distribution. The degree of comparability (see section 2) can be controlled by changing the correlation between the attributes: a strong positive correlation gives high comparability, and a strong negative correlation gives low comparability. We determine the partial order on the generated data points, and again use the algorithm described in section 4 to generate a random

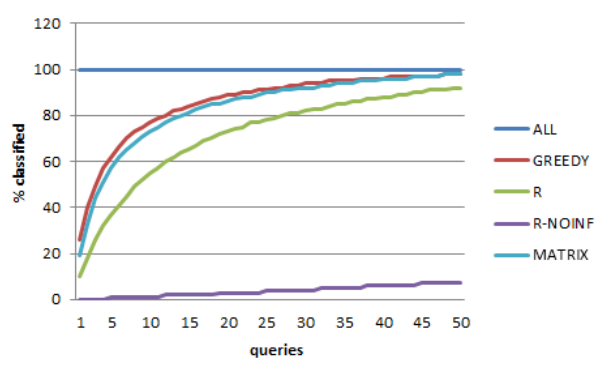

(a) \% labeled vectors

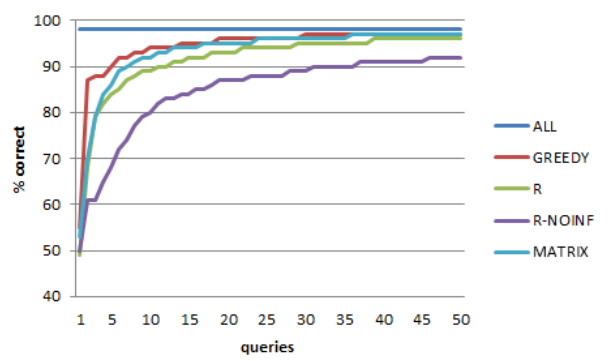

(b) accuracy on test set

Figure 1: Average results on 100 artificial data sets $(k=2, m=50, d=0.5)$

monotone classification on the order concerned.

An interesting phenomenon occurs as the number of class labels increases (see Table 5 and Figure 2). The RANDOM strategy actually starts to outperform the GREEDY heuristic. For example, for $k=7$, after 5 queries the model produced by GREEDY classifies $18 \%$ of the test set correctly, against $28 \%$ for RANDOM. In the end GREEDY catches up, but it is never better. The problem with GREEDY in case there are many class labels is that initially it is not able to uniquely determine the class label of sufficiently many data points, leading to relatively small training samples. In Figure 2(a) we can see that after about 15 queries GREEDY has catched up, but in any case the number of inferred class labels of GREEDY and RANDOM remain very close. However, if we also include points into the training sample whose label has not been uniquely determined yet, but whose label set has been reduced by at least $50 \%$ (indicated by PARTIAL), then GREEDY regains its edge (see Table 5 and Figure 2(c)).

Finally, we consider the effect of the degree of comparability on the performance of the different query strategies. In Figure 3 the degree of comparability has been plotted on the x-axis. The number of queries has been fixed at seven in this case. First of all, we note that the performance increases with the degree of comparability of the data for all query strategies. This was to be expected, since the more comparable 


\begin{tabular}{|l|l|l|l|l||l|l|l|l|}
\cline { 2 - 10 } \multicolumn{1}{c|}{} & \multicolumn{4}{c||}{$m=50, d=0.2$} & \multicolumn{4}{c|}{$m=50, d=0.5$} \\
\hline \% labeled & $20 \%$ & $50 \%$ & $80 \%$ & $100 \%$ & $20 \%$ & $50 \%$ & $80 \%$ & $100 \%$ \\
\hline \hline GREEDY & 1 & 3 & 11 & 45 & 1 & 3.1 & 12 & 67 \\
\hline GBS & 2.1 & 3.8 & 13.4 & 42 & 2.1 & 3.8 & 14 & 56 \\
\hline RANDOM & 1.1 & 8 & 23 & 56 & 1.1 & 8.3 & 27 & 95 \\
\hline
\end{tabular}

Table 4: Average number of queries needed to classify stated \% of vectors averaged over 100 repetitions.

\begin{tabular}{|l|l|l|l|l||l|l|l|l|}
\cline { 2 - 9 } \multicolumn{1}{c|}{} & \multicolumn{3}{c||}{$k=5(\max =73 \%)$} & \multicolumn{4}{c|}{$k=7(\max =64 \%)$} \\
\hline$q$ & 5 & 10 & 20 & 50 & 5 & 10 & 20 & 50 \\
\hline \hline GREEDY & $31 \%$ & $56 \%$ & $67 \%$ & $72 \%$ & $18 \%$ & $32 \%$ & $50 \%$ & $60 \%$ \\
\hline RANDOM & $42 \%$ & $56 \%$ & $64 \%$ & $71 \%$ & $28 \%$ & $41 \%$ & $51 \%$ & $60 \%$ \\
\hline GREEDY-PARTIAL & $56 \%$ & $64 \%$ & $70 \%$ & $73 \%$ & $38 \%$ & $47 \%$ & $56 \%$ & $62 \%$ \\
\hline RANDOM-PARTIAL & $50 \%$ & $60 \%$ & $66 \%$ & $71 \%$ & $33 \%$ & $43 \%$ & $53 \%$ & $61 \%$ \\
\hline
\end{tabular}

Table 5: \% of correctly labeled test vectors after stated number of queries averaged over 100 repetitions. max indicates the prediction error when the complete training set is used $(n=200, c=0.5)$.

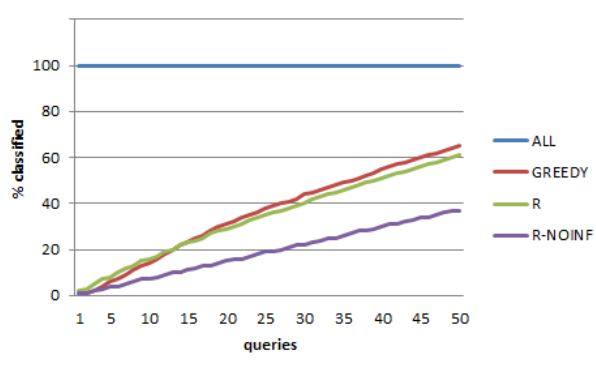

(a) \% labeled vectors

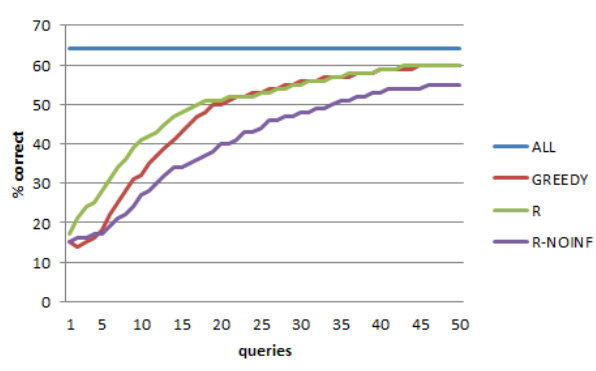

(b) accuracy on test set

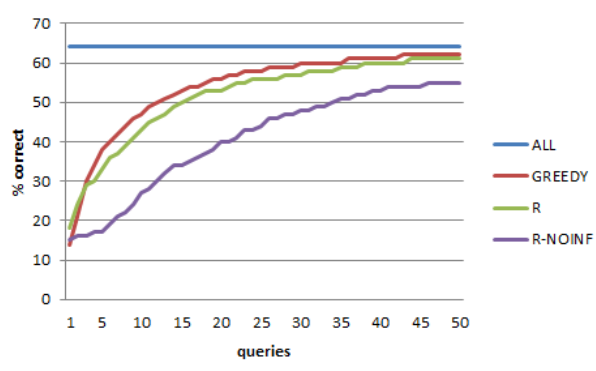

(c) accuracy with partial label information pairs of data points, the higher the benefit of the monotonicity constraint. For example, if there are no comparable pairs at all, the order on the data points is one big anti chain, and the monotonicity constraint has no effect whatsoever. Conversely, if all pairs of points are comparable we have a linear order with maximum benefit of the monotonicity constraint. In figure 3(a) we see that the advantage of GREEDY over RANDOM increases with comparability. If comparability is low there is little scope for inference, and basically every query strategy will perform quite badly. As comparability increases, so does the scope for inference, and well-chosen queries start to pay off.

Comparing Figures 3(b) and 3(c) we see that including partial label information is more useful in data sets with low comparability. This makes perfect sense, since in such data sets there is little scope for inference. Therefore, after a limited number of queries few labels will have been uniquely determined, and the added benefit of partial label information can be substantial.

5.2 Experiments on Real Data In this section we discuss the experiments that we performed on a collection of (almost) real data sets. Their basic properties are summarized in Table 6.

All data sets are available from the UCI machine learning repository [2], except for the Windsor housing data, which is available from the Journal of Applied Econometrics Data Archive ${ }^{1}$ and the Ohsumed data set which is available from the LETOR web site ${ }^{2}$. Monotonicity judgments were based on common sense. For example, in the AutoMPG data, the weight of the

Figure 2: Average results on 100 artificial data sets with $(k=7, n=200, c=0.5)$.

\footnotetext{
${ }^{1}$ http ://econ.queensu.ca/jae/

${ }^{2}$ http://research.microsoft.com/en-us/um/beijing/projects/letor/
} 


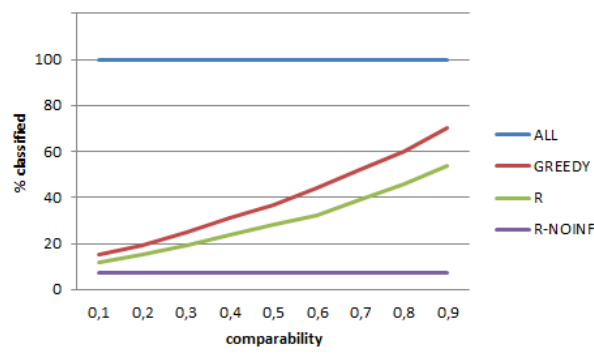

(a) \% labeled vectors

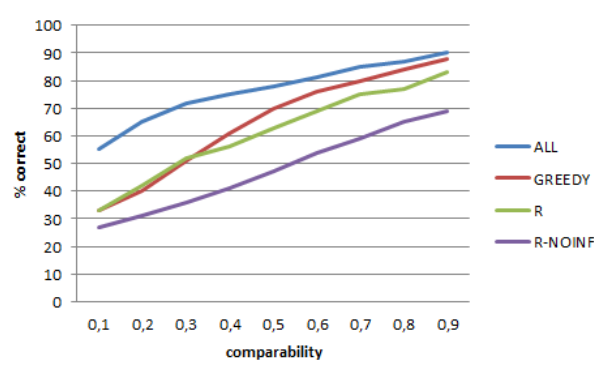

(b) accuracy on test set

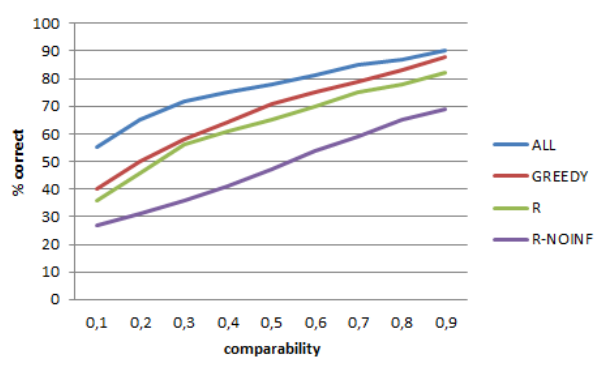

(c) accuracy with partial label information

Figure 3: For each value of $c$ (comparability), the average result of tests on 100 artificial data sets $(k=$ $4, n=200, q=7$ )

\begin{tabular}{|l|l|l|r|l|}
\hline Name & $n$ & $k$ & \#att & $c$ \\
\hline \hline AutoMPG & 392 & 7 & 4 & 0.81 \\
\hline CPU & 209 & 4 & 6 & 0.48 \\
\hline Haberman & 306 & 2 & 3 & 0.33 \\
\hline Windsor & 546 & 4 & 11 & 0.26 \\
\hline Pima & 768 & 2 & 8 & 0.07 \\
\hline Ohsumed & 156 & 2 & 2 & 0.66 \\
\hline
\end{tabular}

Table 6: Basic properties of real data sets after preprocessing; \#att stands for number of attributes and $c$ stands for comparability. car was taken to have a negative influence on miles per gallon, and in the Windsor housing data, lot size was taken to have a positive influence on selling price of the house. The numeric targets of AutoMPG, CPU, and Windsor were discretized into 7, 4, and 4 equal frequency bins respectively. For the Ohsumed data, we selected the data for query 3 , and attributes 1 and 16. To obtain a monotone classification of the data sets we relabeled the data using the algorithm described in [5]. For example, to make the Pima data set monotone, the algorithm relabeled 53 of the 768 data points. Although the Ohsumed data originally had 3 labels ("irrelevant", "partially relevant" and "highly relevant"), after relabeling only 2 labels remained.

After preprocessing, the Ohsumed data set conformed to the restrictions of two class labels and two attributes, and hence we could apply our generalized binary search for matrix orders to it. The results are shown in table 7 . Very few queries are required to infer all class labels. This is explained by the high comparability $(c=0.66)$, and in particular by the fact that the data set contains quite a few identical attribute vectors. Apart from that we observe the same phenomenon as in the artificial data: GREEDY requires fewer queries than GBS on average to infer $80 \%$ of the labels but GBS wins when $100 \%$ of the labels are to be inferred.

\begin{tabular}{|l|l|l|l|l|}
\hline$\%$ labeled & $20 \%$ & $50 \%$ & $80 \%$ & $100 \%$ \\
\hline \hline GREEDY & 1 & 1.2 & 3.2 & 15 \\
\hline GBS & 1 & 1 & 4 & 13 \\
\hline RANDOM & 1 & 2.2 & 6.3 & 24 \\
\hline
\end{tabular}

Table 7: Oshumed: average \# queries needed to classify stated $\%$ of vectors averaged over 100 repetitions.

The results of our experiments on real data are summarized in Table 8 . In general we observe that GREEDY outperforms RANDOM in terms of the number of inferred data points as well as in terms of the accuracy of induced models on the test set. We also see that using partial label information tends to improve the accuracy of induced models. The most extreme example is Windsor housing where after querying 20 data points the accuracy of GREEDY is $60 \%$ and for GREEDY-PARTIAL it's $65 \%$.

\section{Conclusion}

We have shown that monotonicity constraints can be exploited to infer class labels of unlabeled points in ordinal classification problems. We have investigated different query strategies and evaluated their performance. For binary classification we developed an efficient algorithm to count monotone classifications on an incomplete ma- 


\begin{tabular}{|l|l|l|l|l|l|l|l|l|}
\hline Name & Heuristic & $20 \%$ & $50 \%$ & $80 \%$ & 5 & 10 & 20 & $\max$ \\
\hline \hline \multirow{5}{*}{ AutoMPG } & GREEDY & 3.4 & 34 & 74 & $39 \%$ & $60 \%$ & $69 \%$ & $91 \%$ \\
& RANDOM & 6.2 & 44 & 85 & $34 \%$ & $52 \%$ & $65 \%$ & \\
& GREEDY-PARTIAL & & & & $59 \%$ & $66 \%$ & $71 \%$ & \\
& RANDOM-PARTIAL & & & & $50 \%$ & $60 \%$ & $67 \%$ & \\
\hline \multirow{5}{*}{ CPU } & GREEDY & 7.3 & 31 & 65 & $45 \%$ & $67 \%$ & $69 \%$ & $74 \%$ \\
& RANDOM & 12 & 39 & 79 & $40 \%$ & $52 \%$ & $65 \%$ & \\
& GREEDY-PARTIAL & & & & $59 \%$ & $68 \%$ & $70 \%$ & \\
& RANDOM-PARTIAL & & & & $49 \%$ & $61 \%$ & $66 \%$ & \\
\hline \multirow{2}{*}{ Haberman } & GREEDY & 1.1 & 5.3 & 20 & $85 \%$ & $89 \%$ & $90 \%$ & $91 \%$ \\
& RANDOM & 2 & 8.5 & 25.3 & $84 \%$ & $86 \%$ & $89 \%$ & \\
\hline \multirow{5}{*}{ Windsor } & GREEDY & 17 & 72 & 160 & $44 \%$ & $51 \%$ & $60 \%$ & $72 \%$ \\
& RANDOM & 18 & 93 & 176 & $37 \%$ & $47 \%$ & $56 \%$ & \\
& GREEDY-PARTIAL & & & & $53 \%$ & $60 \%$ & $65 \%$ & \\
& RANDOM-PARTIAL & & & & $44 \%$ & $52 \%$ & $59 \%$ & \\
\hline \multirow{2}{*}{ Pima } & GREEDY & 12 & 116 & 256 & $74 \%$ & $77 \%$ & $78 \%$ & $79 \%$ \\
& RANDOM & 24 & 102 & 222 & $72 \%$ & $75 \%$ & $77 \%$ & \\
\hline \multirow{2}{*}{ Ohsumed } & GREEDY & 1 & 1.2 & 3.2 & $97 \%$ & $98 \%$ & $99 \%$ & $99 \%$ \\
& RANDOM & 1 & 2.2 & 6.3 & $91 \%$ & $97 \%$ & $99 \%$ & \\
\hline
\end{tabular}

Table 8: Test results for the real data sets. All results are averages over 100 train-test partitions, where $\frac{2}{3}$ is used for training. The first three columns of numbers are the number of queries it takes to classify $20 \%, 50 \%$ and $80 \%$ of the training set respectively. The next three columns are the percentages of correctly labeled test vectors after 5, 10 and 20 queries respectively. The final column gives the accuracy on the test set when using all training data.

trix order. This enabled us to compare a well-known active learning heuristic called generalized binary search, to an easy-to-compute greedy heuristic. We found that the greedy heuristic initially performs better, but generalized binary search tends to win if the aim is to classify the whole training set. In the context of active learning this is rarely the case, so we may conclude that the greedy heuristic compares favorably to generalized binary search.

In case of non-binary classification it is rather wasteful to ignore the partial label information inferred from the query points. We have proposed a way to incorporate such partial information into the training sample. The experiments showed that this leads to improved performance. In the experiments on artificial data we found that the greedy heuristic benefited more from the partial label information than the random strategy. The greedy heuristic using partial label information emerged as the overall best strategy on the real data as well.

\section{References}

[1] E.A. Altendorf, A.C. Restificar, and T.G. Dietterich. Learning from sparse data by exploiting monotonicity constraints. In Proceedings of the 21st Conference on Uncertainty in Artificial Intelligence (UAI-05), pages 18-25, 2005.
[2] A. Asuncion and D.J. Newman. UCI machine learning repository, 2007.

[3] N. Barile and A. Feelders. Active learning with monotonicity constraints. In SIAM International Conference on Data Mining (SDM 2012), pages 756-767, 2012.

[4] S. Dasgupta. Analysis of a greedy active learning strategy. In Advances in Neural Information Processing Systems (NIPS), pages 337-344. MIT Press, 2004.

[5] A. Feelders. Monotone relabeling in ordinal classification. In ICDM 2010, Proceedings of the 10th IEEE International Conference on Data Mining, pages 803808, 2010.

[6] W. Kotlowski and R. Slowinski. Rule learning with monotonicity constraints. In Proceedings of the 26th Annual International Conference on Machine Learning (ICML 2009), pages 537-544, 2009.

[7] J. Propp and D. Wilson. Exact sampling with coupled markov chains and applications to statistical mechanics. Random Struct. Algorithms, 9(1-2):223-252, 1996.

[8] J. Provan and M. Ball. The complexity of counting cuts and of computing the probability that a graph is connected. SIAM Journal on Computing, 12(4):777788, 1983.

[9] Pieter Soons and Ad Feelders. Exploiting monotonicity constraints in active learning for ordinal classification. Technical Report UU-CS-2014-001, Department of Information and Computing Sciences, Utrecht University, Utrecht, 2014. 